\title{
Security Reductions of the Second Round SHA-3 Candidates
}

\author{
Elena Andreeva, Bart Mennink and Bart Preneel \\ Dept. Electrical Engineering, ESAT/COSIC and IBBT \\ Katholieke Universiteit Leuven, Belgium \\ \{elena.andreeva, bart.mennink\}@esat.kuleuven.be
}

\begin{abstract}
In 2007, the US National Institute for Standards and Technology announced a call for the design of a new cryptographic hash algorithm in response to vulnerabilities identified in existing hash functions, such as MD5 and SHA-1. NIST received many submissions, 51 of which got accepted to the first round. At present, 14 candidates are left in the second round. An important criterion in the selection process is the SHA-3 hash function security and more concretely, the possible security reductions of the hash function to the security of its underlying building blocks. While some of the candidates are supported with firm security reductions, for most of the schemes these results are still incomplete. In this paper, we compare the state of the art provable security reductions of the second round candidates. We discuss all SHA-3 candidates at a high functional level, and analyze and summarize the security reduction results. Surprisingly, we derive some security bounds from the literature, which the hash function designers seem to be unaware of. Additionally, we generalize the well-known proof of collision resistance preservation, such that all SHA-3 candidates with a suffix-free padding are covered.
\end{abstract}

\section{Introduction}

Hash functions are a building block for numerous cryptographic applications. In 2004 a series of attacks by Wang et al. 51/52] have exposed security vulnerabilities in the design of the most widely adopted and deployed SHA-1 hash function. As a result, the US National Institute for Standards and Technology (NIST) recommended the replacement of SHA-1 by the SHA-2 hash function family and announced a call for the design of a new SHA-3 hashing algorithm. The SHA-3 hash function must allow for message digests of length 224,256,384 and 512 bits, it should be efficient, and most importantly it should provide an adequate level of security. In the current second round, 14 candidate hash functions are still in the race for the selection of the SHA-3 hash function. These candidates are under active evaluation by the cryptographic community. As a result of the performed comparative analysis, several classifications of the SHA-3 candidates, mostly focussed on hardware performance, appeared in the literature [2750|26 35]. A classification based on the specified by NIST security criteria is however still due.

NIST Security Requirements. NIST specifies a number of security requirements 43 , to be satisfied by the future SHA-3 function: (i) at least one variant of the hash function must securely support HMAC and randomized hashing. Furthermore, for all $n$-bit digest values, the hash function must provide (ii) preimage resistance of approximately $n$ bits, (iii) second preimage resistance of approximately $n-L$ bits, where the first preimage is of length at most $2^{L}$ blocks, (iv) collision resistance of approximately $n / 2$ bits, and (v) all variants must be resistant to the length-extension attack. Finally, (vi) for any $m \leq n$, the hash function specified by taking a fixed subset of $m$ bits of the function's output is required to satisfy properties (ii)-(v) with $n$ replaced by $m$.

Our Contribution. In this work we provide a survey of the 14 remaining SHA-3 candidates, in which we compare their security reductions. More concretely, we consider preimage, second preimage and collision resistance (security requirements (ii)-(iv)) for the $n=256$ and $n=512$ variants. Most of our security analysis is realized in the ideal model, where one or more of the underlying 
integral building blocks (e.g., the underlying block cipher or permutation(s)) are assumed to be ideal, i.e. random primitives. Additionally, to argue collision security we extend the standard proof of Merkle-Damgård collision resistance [2141] to cover all SHA-3 candidate hash functions with a suffix-free padding (App. A). Notice that the basic Merkle-Damgård proof does not suffice in the presence of a final transformation and/or a chopping.

Furthermore, we consider the indifferentiability of the candidates. Informally, indifferentiability guarantees that a design has no structural design flaws [20], and in particular (as formally proven in App. B an indifferentiability result provides upper bounds on the advantage of finding preimages, second preimages and collisions.

Our main contribution consists in performing a comparative survey of the existing security results on the 14 hash function candidates and results derivable from earlier works on hash functions, and suggesting possible research directions aimed at resolving some of the identified open problems.

Section 2 briefly covers the notation, and the basic principles of hash function design. In Sect. 3 , we consider all candidates from a provable security point of view. We give a high level algorithmic description of each hash function, and discuss the existing security results. All results are summarized in Table 1. We conclude the paper with Sect. 4 and give some final remarks on the security comparison.

\section{Preliminaries}

For a positive integer value $n \in \mathbb{N}$, we denote by $\mathbb{Z}_{2}^{n}$ the set of bit strings of length $n$, and by $\left(\mathbb{Z}_{2}^{n}\right)^{*}$ the set of strings of length a positive multiple of $n$ bits. We denote by $\mathbb{Z}_{2}^{*}$ the set of bit strings of arbitrary length. If $x, y$ are two bit strings, their concatenation is denoted by $x \| y$. By $|x|$ we denote the length of a bit string $x$, and for $m, n \in \mathbb{N}$ we denote by $\langle m\rangle_{n}$ the encoding of $m$ as an $n$-bit string. The function $\operatorname{chop}_{n}(x)$ chops off $n$ bits of a bit string $x$.

Throughout, we use a unified notation for all candidates. The value $n$ denotes the output size of the hash function, $l$ the size of the chaining value, and $m$ the number of message bits compressed in one iteration of the compression function. A padded message is always parsed as a sequence of $k \geq 1$ message blocks of length $m$ bits: $\left(M_{1}, \ldots, M_{k}\right)$.

\subsection{Security Notions}

In this section we investigate the security of the hash functions in the 'ideal model' and the more classical 'generic' security.

Security in the ideal model. In the ideal model, a compressing function $F$ (either on fixed or arbitrary input lengths) that uses one or more underlying building blocks is viewed insecure if there exists a successful information-theoretic adversary that has only query access to the idealized underlying primitives of $F$. The complexity of the attack is measured by the number of queries $q$ to the primitive made by the adversary. In this work it is clear from the context which of the underlying primitives is assumed to be ideal. The three main security properties required from the SHA-3 hash function are preimage, second preimage and collision resistance. For each of these three notions, with $\mathbf{A} \mathbf{d v}_{F}^{\text {atk }}$, where atk $\in\{$ pre, sec, col $\}$, we denote the maximum advantage of an adversary to break the function $F$ under the security notion atk. The advantage is the probability function taken over all random choices of the underlying primitives, and the maximum is taken over all adversaries that make at most $q$ queries to their oracles.

Additionally, we consider the indifferentiability of the SHA-3 candidates. The indifferentiability 
framework introduced by Maurer et al. [40] is an extension of the classical notion of indistinguishability, and ensures that a hash function has no structural defects. We denote the indifferentiability security of a hash function $\mathcal{H}$ by $\mathbf{A} \mathbf{d v}_{\mathcal{H}}^{\text {pro }}$, maximized over all distinguishers making at most $q$ queries of maximal length $K \geq 0$ message blocks to their oracles. We refer to [20] for a formal definition. An indifferentiability bound guarantees security of the hash function against specific attacks. In particular, one can obtain a bound on $\mathbf{A d v}_{\mathcal{H}}^{\text {atk }}$, for any security notion atk: $\mathbf{A} \mathbf{d} \mathbf{v}_{\mathcal{H}}^{\text {atk }} \leq \mathbf{P r}_{R O}^{\text {atk }}+\mathbf{A d d} \mathbf{v}_{\mathcal{H}}^{\text {pro }}$, where $\mathbf{P r}_{R O}^{\text {atk }}$ denotes the success probability of a generic attack against $\mathcal{H}$ under atk. This bound is proven in Thm. 2 (App. B).

Generic security. The generic collision security in the context of this work deals with analyzing the collision resistance of hash functions in the standard model. A hash function $\mathcal{H}$ is called generically $(t, \varepsilon)$ collision resistant if no adversary running in time at most $t$ can find two different messages $M, M^{\prime}$ such that $\mathcal{H}(M)=\mathcal{H}\left(M^{\prime}\right)$ with advantage more than $\varepsilon$. We denote by $\mathbf{A d v}_{\mathcal{H}}^{\text {gcol }}$ the generic collision resistance security of the function $\mathcal{H}$, maximized over all 'efficient' adversaries. We refer the reader to [46452] for a more formal discussion.

To argue generic collision security of the hash function $\mathcal{H}$ (as domain extenders of fixed input length compression functions) we use the composition result of [2141] and extend it to a wider class of suffix-free hash functions (App. A). This result concludes the collision security of the hash function $\mathcal{H}$ assuming collision security guarantees from the underlying compression functions. We then translate ideal model collision security results on the compression functions via the latter composition to ideal model collision results on the hash function (expressed by $\mathbf{A d} \mathbf{d v}_{\mathcal{H}}^{\text {col }}$ ). A generic collision result, generally speaking, applies to a wider class of schemes for which no bounds on the collision security of the underlying compression functions is known, e.g. for BLAKE and BMW.

If a compressing function $F$ outputs a bit string of length $n$, one expects to find collisions with high probability after approximately $2^{n / 2}$ queries (due to the birthday attack). Similarly, (second) preimages can be found with high probability after approximately $2^{n}$ queries 1 . Moreover, finding second preimages is provably harder than finding collisions, and similar for preimages (conditionally) [46]. Formally, we have $\Omega\left(q^{2} / 2^{n}\right)=\mathbf{A} \mathbf{d v}_{F}^{\text {col }}=O(1), \Omega\left(q / 2^{n}\right)=\mathbf{A} \mathbf{d} \mathbf{v}_{F}^{\text {sec }} \leq \mathbf{A} \mathbf{d} \mathbf{v}_{F}^{\text {col }}$, and $\Omega\left(q / 2^{n}\right)=$ $\mathbf{A} \mathbf{d} \mathbf{v}_{F}^{\text {pre }} \leq \mathbf{A} \mathbf{d} \mathbf{v}_{F}^{\text {col }}+\varepsilon$, where $\varepsilon$ is negligible if $F$ is a variable input length compressing function. In the remainder, we will consider these bounds for granted, and only include security results that improve either of these bounds. A bound is called tight if the lower and upper bound are the same up to a constant factor, and optimal if the bound is tight with respect to the original lower bound.

\subsection{Compression Function Design Strategies}

A common way to build compression functions is to base it on a block cipher [44]1649], or on a (limited number of) permutation(s) [15,47/48]. Preneel et al. 44] analyzed and categorized 64 block cipher based compression functions. Twelve of them are formally proven secure by Black et al. [16. These results have been recently generalized by Stam [49]. Interestingly, the latter result implies security bounds for some compression functions that do not fit in the PGV-model, like ECHO, Hamsi and SIMD. Throughout, by 'PGVx' we denote the $x^{\text {th }}$ type compression function of [44. We note that PGV1, PGV3 and PGV5 are better known as the Matyas-Meyer-Oseas, the Miyaguchi-Preneel and the Davies-Meyer compression functions, respectively.

\footnotetext{
${ }^{1}$ Kelsey and Schneier [34] describe a second preimage attack on the Merkle-Damgård hash function that requires at most approximately $2^{n-L}$ queries, where the first preimage is of length at most $2^{L}$ blocks. This attack does, however, not apply to all SHA-3 candidates. In particular, wide-pipe designs remain mostly unaffected due to their increased internal state 34 .
} 
In the context of permutation based compression functions, Black et al. [15] analyzed $2 l$ - to $l$-bit compression functions based on one $l$-bit permutation, and proved them insecure. This result has been generalized by Rogaway and Steinberger [47] and Stam [48] to compression functions with arbitrary input and output sizes, and an arbitrary number of underlying permutations. Their bounds indicate the expected number of queries required to find collisions or preimages for permutation based compression functions.

\subsection{Hash Function Design Strategies}

In order to allow the hashing of arbitrarily long strings, all SHA-3 candidates employ a specific mode of operation. Central to all designs is the iterated hash function principle [37]: on input of an initialization vector IV, the iterated hash function $\mathcal{H}^{f}$ based on the compression function $f$ proceeds a padded message $\left(M_{1}, \ldots, M_{k}\right)$ as follows:

$$
\begin{aligned}
\mathcal{H}^{f}\left(\mathrm{IV} ; M_{1}, \ldots, M_{k}\right)=h_{k}, \text { where: } h_{0} & =\mathrm{IV}, \\
h_{i} & =f\left(h_{i-1}, M_{i}\right) \text { for } i=1, \ldots, k .
\end{aligned}
$$

This principle is also called the plain Merkle-Damgård (MD) design 4121]. Each of the 14 remaining candidates is based on this design, possibly followed by a final transformation (FT), and/or a chop-function ${ }^{2}$.

The padding function pad : $\mathbb{Z}_{2}^{*} \rightarrow\left(\mathbb{Z}_{2}^{m}\right)^{*}$ is an injective mapping that transforms a message of arbitrary length to a message of length a multiple of $m$ bits (the number of message bits compressed in one compression function iteration). Most of the candidates employ a sufficiently strong padding rule (cf. App. C). Additionally, in some of the designs the message blocks are compressed along with specific counters or tweaks, which may strengthen the padding rule. We distinguish between 'prefix-free' and/or 'suffix-free' padding.

A padding rule is called suffix-free, if for any distinct $M, M^{\prime}$, there exists no bit string $X$ such that $\operatorname{pad}\left(M^{\prime}\right)=X \| \operatorname{pad}(M)$. The plain MD design with any suffix-free padding (also called MD-strengthening [37]) preserves collision resistance [4121]. We generalize this result in Thm. 1 (App. A): informally, this preservation result also holds if the iteration is finalized by a distinct compression function and/or the chop-function. Other security properties, like preimage resistance, are however not preserved in the MD design [2. It is also proven that the MD design with a suffix-free padding need not necessarily be indifferentiable [20]. However, the MD construction is indifferentiable if it ends with a chopping function or a final transformation $[20]^{3}$.

A padding rule is called prefix-free, if for any distinct $M, M^{\prime}$, there exists no bit string $X$ such that $\operatorname{pad}\left(M^{\prime}\right)=\operatorname{pad}(M) \| X$. It has been proved in [20] that the MD design with prefix-free padding is indifferentiable from a random oracle. Security notions like collision-resistance, are however not preserved in the MD design with prefix-free only padding.

HAIFA design. A concrete design based on the MD principle is the HAIFA construction [13]. In HAIFA the message is padded in a specific way so as to solve some deficiencies of the original MD construction: in the iteration, each message block is accompanied with a fixed (optional) salt of $s$ bits and a (mandatory) counter $C_{i}$ of $t$ bits. The counter $C_{i}$ keeps track of the number of message

\footnotetext{
${ }^{2}$ A function $g$ is a final transformation if it differs from $f$, and is applied to the final state, possibly with the injection of an additional message block. The chop-function is not considered to be (a part of) a final transformation.

3 The indifferentiability results of [20] hold if the underlying compression function is ideal, or if the hash function is based on the PGV5 construction with ideal block cipher.
} 
bits hashed so far, and equals 0 by definition if the $i^{\text {th }}$ block does not contain any message bits. Partially due to the properties of this counter, the HAIFA padding rule is suffix- and prefix-free. As a consequence, the construction preserves collision resistance (cf. Thm. 1) and the indifferentiability results of [20] carry over. For the HAIFA design, these indifferentiability results are improved in [11. Furthermore, the HAIFA construction is proven secure against second preimage attacks if the underlying compression function is assumed to behave like an ideal primitive [18].

Wide-pipe design. In the wide-pipe design [39, the iterated state size is significantly larger than the final hash output: at the end of the iteration, a fraction of the output of a construction is discarded. As proved in 20, the MD construction with a distinct final transformation and/or chopping at the end is indifferentiable from a random oracle.

Sponge functions. We do not explicitly consider sponge functions [10] as a specific type of construction: all SHA-3 candidates known to be sponge(-like) functions, CubeHash, Fugue, JH, Keccak and Luffa, can be described in terms of the chop-MD construction (possibly with a final transformation before or instead of the chopping).

\section{SHA-3 Hash Function Candidates}

In this section, we analyze the security of the 14 remaining SHA-3 candidates in more detail. For simplicity, we only consider the proposals of the SHA-3 candidates that output digests of 256 or 512 bits. Observe that in many candidate SHA-3 hash function families, the algorithms that output 224 or 384 bits are the same as the 256- or 512-bits algorithms, except for an additional chopping at the end. Particularly, the results of [20] and Thm. 1 carry over in most of the cases. The same remark applies to requirement (vi) of NIST.

Requirement (i). All designers claim that their proposal can safely be used in HMAC mode [4] or for randomized hashing [33], and we do not discuss it here;

Requirements (ii)-(iv). Preimage, second preimage and collision resistance of each hash function are discussed in this section. Additionally, we consider the indifferentiability of the candidates;

Requirement (v). All hash function candidates are secure against the length extension attack, and thus we do not discuss it further.

Below, we examine the SHA-3 candidate hash functions in more detail. Each paragraph contains an informal discussion for each of the second round SHA-3 candidates and their security reduction results. The mathematical descriptions of the (abstracted) designs are given in Fig. 1, and the candidates' padding functions are summarized in App. C. The concrete security results for all current candidate hash functions are summarized in Table 1. More precisely, for each candidate and each security notion, this table includes the security bound, as far as it exists, and the underlying assumption.

3.1. The BLAKE hash function [3] is a HAIFA construction. The message blocks are accompanied with a HAIFA-counter, and more generally, the function employs a suffix- and prefix-free padding rule. The compression function $f$ is block cipher based 4 . It moreover employs an injective linear function $L$, and a linear function $L^{\prime}$ that XORs the first and second halves of the input.

Security of BLAKE. The compression function of BLAKE shows similarities with the PGV5 compression function [16], but no security results are known for this variation. The mode of operation

\footnotetext{
${ }^{4}$ As observed in [3, Sect. 5], the core part of the compression function can be seen as a permutation keyed by the message, which we view here as a block cipher.
} 


\begin{tabular}{|c|c|}
\hline \multirow[t]{2}{*}{$\begin{array}{l}\text { BLAKE: } \\
(n, l, m, s, t) \in\{(256,256,512,128,64), \\
\quad(512,512,1024,256,128)\} \\
E: \mathbb{Z}_{2}^{2 l} \times \mathbb{Z}_{2}^{m} \rightarrow \mathbb{Z}_{2}^{2 l} \text { a block cipher } \\
L: \mathbb{Z}_{2}^{l+s+t} \rightarrow \mathbb{Z}_{2}^{2 l}, L^{\prime}: \mathbb{Z}_{2}^{2 l} \rightarrow \mathbb{Z}_{2}^{l} \text { linear functions } \\
f(h, M, S, C)=L^{\prime}\left(E_{M}(L(h, S, C))\right) \oplus h \oplus(S \| S)\end{array}$} & $\begin{array}{l}\text { JH: } \\
(n, l, m) \in\{(256,1024,512),(512,1024,512)\} \\
P: \mathbb{Z}_{2}^{l} \rightarrow \mathbb{Z}_{2}^{l} \text { a permutation } \\
f(h, M)=P\left(h \oplus\left(0^{l-m} \| M\right)\right) \oplus\left(M \| 0^{l-m}\right)\end{array}$ \\
\hline & \multirow{2}{*}{$\begin{array}{l}\mathrm{JH}(M)=h, \text { where: } \\
\quad\left(M_{1}, \ldots, M_{k}\right) \leftarrow \operatorname{pad}_{8}(M) ; h_{0} \leftarrow \mathrm{IV} \\
\quad h_{i} \leftarrow f\left(h_{i-1}, M_{i}\right) \text { for } i=1, \ldots, k \\
h \leftarrow \operatorname{chop}_{l-n}\left[h_{k}\right]\end{array}$} \\
\hline \multirow{2}{*}{$\begin{array}{l}\text { BLAKE }(M)=h_{k}, \text { where: } \\
\quad\left(M_{1}, \ldots, M_{k}\right) \leftarrow \operatorname{pad}_{1}(M) ; h_{0} \leftarrow \mathrm{IV} \\
S \in \mathbb{Z}_{2}^{s} ;\left(C_{i}\right)_{i=1}^{k} \text { HAIFA-counter } \\
h_{i} \leftarrow f\left(h_{i-1}, M_{i}, S, C_{i}\right) \text { for } i=1, \ldots, k \\
\end{array}$} & \\
\hline & \multirow{2}{*}{$\begin{array}{l}\text { Keccak: } \\
(n, l, m) \in\{(256,1600,1088),(512,1600,576)\} \\
P: \mathbb{Z}_{2}^{l} \rightarrow \mathbb{Z}_{2}^{l} \text { a permutation } \\
f(h, M)=P\left(h \oplus\left(M \| 0^{l-m}\right)\right)\end{array}$} \\
\hline \multirow{2}{*}{ 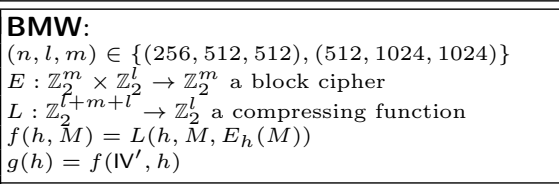 } & \\
\hline & \multirow{2}{*}{$\begin{array}{l}\text { Keccak }(M)=h, \text { where: } \\
\quad\left(M_{1}, \ldots, M_{k}\right) \leftarrow \operatorname{pad}_{9}(M) ; h_{0} \leftarrow \mathrm{IV} \\
\quad h_{i} \leftarrow f\left(h_{i-1}, M_{i}\right) \text { for } i=1, \ldots, k \\
h \leftarrow \operatorname{chop}_{l-n}\left[h_{k}\right] \\
\end{array}$} \\
\hline \multirow{2}{*}{$\begin{array}{l}\text { BMW }(M)=h, \text { where: } \\
\quad\left(M_{1}, \ldots, M_{k}\right) \leftarrow \operatorname{pad}_{2}(M) ; h_{0} \leftarrow \text { IV } \\
\quad h_{i} \leftarrow f\left(h_{i-1}, M_{i}\right) \text { for } i=1, \ldots, k \\
h \leftarrow \operatorname{chop}_{l-n}\left[g\left(h_{k}\right)\right]\end{array}$} & \\
\hline & \multirow{2}{*}{$\begin{array}{l}\text { Luffa: } \\
(n, l, m, w) \in\{(256,768,256,3),(512,1278,256,5)\} \\
P_{i}: \mathbb{Z}_{2}^{m} \rightarrow \mathbb{Z}_{2}^{m}(i=1, \ldots, w) \text { permutations } \\
L: \mathbb{Z}_{2}^{w m+m} \rightarrow \mathbb{Z}_{2}^{w m}, L^{\prime}: \mathbb{Z}_{2}^{w m} \rightarrow \mathbb{Z}_{2}^{m} \text { linear functions } \\
f(h, M)=\left(P_{1}\left(h_{1}^{\prime}\right)\|\cdots\| P_{w}\left(h_{w}^{\prime}\right)\right) \\
\quad \text { where }\left(h_{1}^{\prime}, \ldots, h_{w}^{\prime}\right)=L(h, M) \\
g(h)=\left(L^{\prime}(h) \| L^{\prime}\left(f\left(h, 0^{m}\right)\right)\right)\end{array}$} \\
\hline \multirow{2}{*}{$\begin{array}{l}\text { CubeHash: } \\
(n, l, m) \in\{(256,1024,256),(512,1024,256)\} \\
P: \mathbb{Z}_{2}^{l} \rightarrow \mathbb{Z}_{2}^{l} \text { a permutation } \\
f(h, M)=P\left(h \oplus\left(M \| 0^{l-m}\right)\right) \\
g(h)=P^{10}\left(h \oplus\left(0^{992}\|1\| 0^{31}\right)\right) \\
\end{array}$} & \\
\hline & \multirow{2}{*}{$\begin{array}{l}\text { Luffa }(M)=h, \text { where: } \\
\quad\left(M_{1}, \ldots, M_{k}\right) \leftarrow \operatorname{pad}_{10}(M) ; h_{0} \leftarrow \text { IV } \\
\quad h_{i} \leftarrow f\left(h_{i-1}, M_{i}\right) \text { for } i=1, \ldots, k \\
\quad h \leftarrow \operatorname{chop}_{512-n}\left[g\left(h_{k}\right)\right]\end{array}$} \\
\hline \multirow{2}{*}{$\begin{array}{l}\text { CubeHash }(M)=h, \text { where: } \\
\quad\left(M_{1}, \ldots, M_{k}\right) \leftarrow \operatorname{pad}_{3}(M) ; h_{0} \leftarrow \mathrm{IV} \\
h_{i} \leftarrow f\left(h_{i-1}, M_{i}\right) \text { for } i=1, \ldots, k \\
h \leftarrow \operatorname{chop}_{l-n}\left[g\left(h_{k}\right)\right] \\
\end{array}$} & \\
\hline & \multirow[b]{2}{*}{$\begin{array}{l}\text { Shabal: } \\
(n, l, m) \in\{(256,1408,512),(512,1408,512)\} \\
E: \mathbb{Z}_{2}^{896} \times \mathbb{Z}_{2}^{1024} \rightarrow \mathbb{Z}_{2}^{896} \text { a block cipher } \\
f(h, C, M)=\left(y_{1}, h_{3}-M, y_{3}\right) \\
\quad \text { where } h \in \mathbb{Z}_{2}^{l} \rightarrow h=\left(h_{1}, h_{2}, h_{3}\right) \in \mathbb{Z}_{2}^{384+m+m} \\
\quad \text { and }\left(y_{1}, y_{3}\right)=E_{M, h_{3}}\left(h_{1} \oplus\left(0^{320} \| C\right), h_{2}+M\right)\end{array}$} \\
\hline \multirow{2}{*}{$\begin{array}{l}\text { ECHO: } \\
(n, l, m, s, t) \in\{(256,512,1536,128,64 / 128) \\
\quad(512,1024,1024,256,64 / 128)\} \\
E: \mathbb{Z}_{2}^{2048} \times \mathbb{Z}_{2}^{s+t} \rightarrow \mathbb{Z}_{2}^{2048} \text { a block cipher } \\
L: \mathbb{Z}_{2}^{2048} \rightarrow \mathbb{Z}_{2}^{l} \text { a linear function } \\
f(h, M, S, C) \stackrel{L}{=}\left(E_{S, C}(h \| M) \oplus(h \| M)\right) \\
\end{array}$} & \\
\hline & \multirow{2}{*}{$\begin{array}{l}\text { Shabal }(M)=h, \text { where: } \\
\quad\left(M_{1}, \ldots, M_{k}\right) \leftarrow \operatorname{pad}_{11}(M) ; h_{0} \leftarrow \text { IV } \\
\quad h_{i} \leftarrow f\left(h_{i-1},\langle i\rangle_{64}, M_{i}\right) \text { for } i=1, \ldots, k \\
h_{k+i} \leftarrow f\left(h_{k+i-1},\langle k\rangle_{64}, M_{k}\right) \text { for } i=1, \ldots, 3 \\
h \leftarrow \operatorname{chop}_{l-n}\left[h_{k+3}\right]\end{array}$} \\
\hline \multirow{2}{*}{$\begin{array}{l}\text { ECHO }(M)=h, \text { where: } \\
\quad\left(M_{1}, \ldots, M_{k}\right) \leftarrow \operatorname{pad}_{4}(M) ; h_{0} \leftarrow \mathrm{IV} \\
\quad S \in \mathbb{Z}_{2}^{s} ;\left(C_{i}\right)_{i=1}^{k} \text { HAIFA-counter } \\
h_{i} \leftarrow f\left(h_{i-1}, M_{i}, S, C_{i}\right) \text { for } i=1, \ldots, k \\
h \leftarrow \operatorname{chop}_{l-n}\left[h_{k}\right] \\
\end{array}$} & \\
\hline & \multirow{2}{*}{ 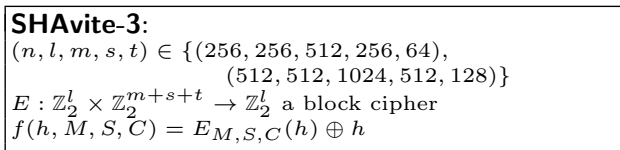 } \\
\hline \multirow{2}{*}{$\begin{array}{l}\text { Fugue: } \\
(n, l, m) \in\{(256,960,32),(512,1152,32)\} \\
P, \tilde{P}: \mathbb{Z}_{2}^{l} \rightarrow \mathbb{Z}_{2}^{l} \text { permutations } \\
L: \mathbb{Z}_{2}^{l} \times \mathbb{Z}_{2}^{m} \rightarrow \mathbb{Z}_{2}^{l} \text { a linear function } \\
f(h, M)=P(L(h, M)) \\
\end{array}$} & \\
\hline & \\
\hline \multirow{2}{*}{$\begin{array}{l}\text { Fugue }(M)=h, \text { where: } \\
\quad\left(M_{1}, \ldots, M_{k}\right) \leftarrow \operatorname{pad}_{5}(M) ; h_{0} \leftarrow \mathrm{IV} \\
\quad h_{i} \leftarrow f\left(h_{i-1}, M_{i}\right) \text { for } i=1, \ldots, k \\
h \leftarrow \operatorname{chop}_{l-n}\left[\tilde{P}\left(h_{k}\right)\right] \\
\end{array}$} & $\begin{array}{l}S \in \mathbb{Z}_{2}^{S} ;\left(C_{i}\right)_{i=1}^{k} \text { HAIFA-counter } \\
h_{i} \leftarrow f\left(h_{i-1}, M_{i}, S, C_{i}\right) \text { for } i=1, \ldots, k \\
\end{array}$ \\
\hline & \multirow{2}{*}{$\begin{array}{l}\text { SIMD: } \\
(n, l, m) \in\{(256,512,512),(512,1024,1024)\} \\
E, \tilde{E}: \mathbb{Z}_{2}^{l} \times \mathbb{Z}_{2}^{m} \rightarrow \mathbb{Z}_{2}^{l} \text { block ciphers } \\
f(h, M)=L\left(h, E_{M}(h \oplus M)\right) \\
g(h, M)=L\left(h, \tilde{E}_{M}(h \oplus M)\right)\end{array}$} \\
\hline \multirow{2}{*}{$\begin{array}{l}\text { Grøstl: } \\
(n, l, m) \in\{(256,512,512),(512,1024,1024)\} \\
P, Q: \mathbb{Z}_{2}^{l} \rightarrow \mathbb{Z}_{2}^{l} \text { permutations } \\
f(h, M)=P(h \oplus M) \oplus Q(M) \oplus h \\
g(h)=P(h) \oplus h\end{array}$} & \\
\hline & \multirow{2}{*}{$\begin{array}{l}\operatorname{SIMD}(M)=h, \text { where: } \\
\quad\left(M_{1}, \ldots, M_{k}\right) \leftarrow \operatorname{pad}_{13}(M) ; h_{0} \leftarrow \text { IV } \\
h_{i} \leftarrow f\left(h_{i-1}, M_{i}\right) \text { for } i=1, \ldots, k-1 \\
h_{k} \leftarrow g\left(h_{k-1}, M_{k}\right) \\
h \leftarrow \operatorname{chop}_{l-n}\left[h_{k}\right] \\
\end{array}$} \\
\hline $\begin{array}{l}M)=h, \mathrm{w} \\
\left.\ldots, M_{k}\right) \\
f\left(h_{i-1},\right. \\
\operatorname{chop}_{l-n}[g\end{array}$ & \\
\hline \multirow{2}{*}{$\begin{array}{l}\text { Hamsi: } \\
(n, l, m) \in\{(256,256,32),(512,512,64)\} \\
P, \tilde{P}: \mathbb{Z}_{2}^{2 n} \rightarrow \mathbb{Z}_{2}^{2 n} \text { permutations } \\
\text { Exp : } \mathbb{Z}_{2}^{m} \rightarrow \mathbb{Z}_{2}^{n} \text { a linear code } \\
f(h, M)=h \oplus \operatorname{chop}_{n}[P(\operatorname{Exp}(M) \| h)] \\
g(h, M)=h \oplus \operatorname{chop}_{n}[\tilde{P}(\operatorname{Exp}(M) \| h)] \\
\end{array}$} & $\begin{array}{l}(n, l, m) \in\{(256,51 \\
E: \mathbb{Z}_{2}^{m} \times \mathbb{Z}_{2}^{128} \times \mathbb{Z}_{2}^{l} \\
f(h, T, M) \stackrel{=}{=} E_{h, T}\end{array}$ \\
\hline & \multirow{2}{*}{$\begin{array}{l}\text { Skein }(M)=h, \text { where: } \\
\quad\left(M_{1}, \ldots, M_{k}\right) \leftarrow \operatorname{pad}_{14}(M) ; h_{0} \leftarrow \mathrm{IV} \\
\quad\left(T_{i}\right)_{i=1}^{k} \text { round-specific tweaks } \\
\quad h_{i} \leftarrow f\left(h_{i-1}, T_{i}, M_{i}\right) \text { for } i=1, \ldots, k \\
h \leftarrow \operatorname{chop}_{l-n}\left[h_{k}\right]\end{array}$} \\
\hline $\begin{array}{l}\operatorname{amsi}(M)=h, \text { where: } \\
\left(M_{1}, \ldots, M_{k}\right) \leftarrow \text { pac } \\
h_{i} \leftarrow f\left(h_{i-1}, M_{i}\right) \mathrm{f}\end{array}$ & \\
\hline
\end{tabular}

Fig. 1. The padding rules employed by the functions are summarized in App. C. In all algorithm descriptions, IV denotes an initialization vector, $h$ denotes state values, $M$ denotes message blocks, $S$ denotes a (fixed) salt, $C$ denotes a counter and $T$ denotes a tweak. The functions $L, L^{\prime}$, Exp underlying BLAKE, BMW, ECHO, Fugue, Hamsi and Luffa, are explained in the corresponding section. 
of BLAKE is based on the HAIFA structure, and as a consequence all security properties regarding this type hold [13. In particular, the design preserves collision resistance, and is secure against second preimage attacks. Also, the BLAKE hash function is indifferentiable from a random oracle if the underlying compression function is assumed to be ideal, due to the HAIFA counter [20 11]. Using Thm. 2, this indifferentiability bound additionally renders an optimal collision resistance bound for BLAKE, $\mathbf{A d v}_{H}^{\mathrm{col}}=\Theta\left(q^{2} / 2^{n}\right)$.

3.2. The Blue Midnight Wish (BMW) hash function [31] is a chop-MD construction, with a final transformation before chopping. The hash function employs a suffix-free padding rule. The compression function $f$ is block cipher based 5 and the final transformation $g$ consists of the same compression function with the chaining value processed as a message, and with an initial value as chaining input. The compression function employs a function $L$ which consists of two compression functions with specific properties as specified in [31].

Security of BMW. The compression function of BMW shows similarities with the PGV3 compression function [16, but no security results are known for this variation. Thm. 11 applies to BMW, where the final transformation has no message block as input. Furthermore, albeit no indifferentiability proof for the BMW hash function is known, we note that BMW can be seen as a combination of the HMAC- and the chop-construction, both proven indifferentiable from a random oracle [20].

3.3. The CubeHash hash function [7] is a chop-MD construction, with a final transformation before chopping. The compression function $f$ is permutation based, and the final transformation $g$ consists of flipping a certain bit in the state and applying 10 more compression function rounds on zero-messages.

Security of CubeHash. The compression function of CubeHash is based on one permutation ${ }^{6}$, and collisions and preimages for the compression function can be found in one query to the permutation [15]. The final transformation of CubeHash consists of ten compression function rounds with zeromessages, preceded by one bit flip in the chaining. As a consequence, throughout the execution of CubeHash, in total at most $m+1$ out of $l$ wires of the chaining are affected by message injection. Therefore this construction follows the sponge design with 'rate' $m+1$, and 'capacity' $l-m-1$, and the indifferentiability result of [8] carries over 7 . Using Thm. 2, this indifferentiability bound additionally renders an optimal collision resistance bound for CubeHash, $\mathbf{A d v}_{H}^{\mathrm{col}}=\Theta\left(q^{2} / 2^{n}\right)$, as well as an improved upper bound $O\left(\frac{q}{2^{n}}+\frac{q^{2}}{2^{l-n}}\right)$ on the preimage and second preimage resistance. Note that these bounds are optimal for the $n=256$ variant.

3.4. The ECHO hash function [6] is a chop-HAIFA construction. The message blocks are accompanied with a HAIFA-counter, and more generally, the function employs a suffix- and prefix-free padding rule. The compression function $f$ is block cipher based ${ }^{8}$. It moreover employs a linear function $L$ that chops the state in blocks of length $l$ bits, and XORs these.

Security of ECHO. The compression function of ECHO is a 'chopped single call Type-I' compression function in the categorization of [49]. Therefore, the results of [49, Thm. 15] carry over, yielding optimal security bounds for the compression function. Observe that these results can easily be ad-

\footnotetext{
${ }^{5}$ As observed in 31, the compression function can be seen as a generalized PGV3 construction, where the function $f_{0}$ of 31 defines the block cipher keyed with the chaining value.

${ }^{6}$ Effectively, this permutation consists of one simpler permutation executed 16 times iteratively.

7 The sponge design requires the last block of a padded message to be non-zero, which is not the case for CubeHash. However, if the squeezing of the sponge takes only one round, this is not required for the indifferentiability proof.

${ }^{8}$ As observed in [6], the core part of the compression function can be seen as a permutation keyed by the salt and counter, which we view here as a block cipher. This cipher is AES-based.
} 
justed to obtain bound $\mathbf{A} \mathbf{d} \mathbf{v}_{\text {chop } \circ f}^{\text {col }}=\Theta\left(q^{2} / 2^{n}\right)$. ECHO is a combination of HAIFA and chop-MD, but it is unclear whether all HAIFA security properties hold after chopping. Still, Thm. 1 applies to $\mathrm{ECHO}$, and as a consequence we obtain $\mathbf{A d} \mathbf{d v}_{\mathcal{H}}^{\mathrm{col}}=\Theta\left(q^{2} / 2^{n}\right)$. Furthermore, the ECHO hash function would be indifferentiable from a random oracle if the underlying compression function is assumed to be ideal, due to the chopping function at the end [20. However, the compression function of ECHO is easily differentiable from a random oracle [29], and we cannot directly apply the results of $[20]$.

3.5. The Fugue hash function 32 is a chop-MD construction, with a final transformation before chopping. The hash function employs a suffix-free padding rule. The compression function $f$ is permutation based, and the final transformation consists of a permutation $\tilde{P}$ which differs from $P$ in the parametrization. The compression function employs a linear function $L$ for message injection (TIX of [32]).

Security of Fugue. The compression function of Fugue is based on one permutation, and collisions and preimages for the compression function can be found in one query to the permutation [15]. As a consequence, the result of Thm. 1 is irrelevant, even though the padding rule of Fugue is suffix-free. The Fugue hash function borrows characteristics from the sponge design, and ideas from the indifferentiability proof of [8] may carry over. This is, however, not immediately clear due to the final transformation before chopping.

3.6. The Grøstl hash function 30 is a chop-MD construction, with a final transformation before chopping. The hash function employs a suffix-free padding rule. The compression function $f$ is permutation based, and the final transformation $g$ is defined as $g(h)=P(h) \oplus h$.

Security of Grøstl. The compression function of Grøstl is permutation based, and the results of 47/48 apply. Furthermore, the preimage resistance of the compression function is analyzed in [28], and an upper bound for collision resistance can be obtained easily. As a consequence, we obtain tight security bounds on the compression function, $\mathbf{A d} \mathbf{d v}_{f}^{\text {pre }}=\Theta\left(q^{2} / 2^{l}\right)$ and $\mathbf{A d v}_{f}^{\text {col }}=\Theta\left(q^{4} / 2^{l}\right)$. Thm. 1 applies to Grøstl, where the final transformation has no message block as input. Observe that we also have $\mathbf{A} \mathbf{d} \mathbf{v}_{\text {chopog }}^{\text {col }}=\Theta\left(q^{2} / 2^{n}\right)$, and as a consequence we obtain $\mathbf{A d v}_{\mathcal{H}}^{\text {col }}=\Theta\left(q^{2} / 2^{n}\right)$. Furthermore, the Grøstl hash function is proven indifferentiable from a random oracle if the underlying permutations are ideal [1].

3.7. The Hamsi hash function [36] is a MD construction, with a final transformation before chopping. The hash function employs a suffix-free padding rule. The compression function $f$ is permutation based, but the last round is executed with a compression function $g$ based on a permutation $\tilde{P}$ which differs from $P$ in the parametrization. The compression functions employ a linear code Exp for message injection [36].

Security of Hamsi. The compression function of Hamsi is a 'chopped single call Type-I' compression function in the categorization of [49]. Therefore, the results of [49, Thm. 15] carry over, yielding optimal security bounds for the compression function. Observe that these bounds also apply to the function $g$. Thm. 1 applies to Hamsi, and as a consequence we obtain $\mathbf{A d v}_{\mathcal{H}}^{\text {col }}=\Theta\left(q^{2} / 2^{n}\right)$. Furthermore, albeit no indifferentiability proof for the Hamsi hash function is known, we note that Hamsi can be seen as a variation of the NMAC-construction, which is proven indifferentiable from a random oracle [20].

3.8. The $\mathbf{J H}$ hash function [53] is a chop-MD construction. The hash function employs a suffix-free padding rule. The compression function $f$ is permutation based.

Security of $\mathbf{J H}$. The compression function of $\mathbf{J H}$ is based on one permutation, and collisions and 
preimages for the compression function can be found in one query to the permutation [15]. As a consequence, the result of Thm. 1 is irrelevant, even though the padding rule of JH is suffix-free. The JH hash function is proven indifferentiable from a random oracle if the underlying permutation is assumed to be ideal [12. Using Thm. 2, this indifferentiability bound additionally renders improved upper bounds on the preimage, second preimage and collision resistance: we obtain $\mathbf{A} \mathbf{d} \mathbf{v}_{\mathcal{H}}^{\text {col }}=O\left(\frac{q^{2}}{2^{n}}+\frac{q^{3}}{2^{l-m}}\right)$, and both $\mathbf{A d v}_{\mathcal{H}}^{\text {pre }}$ and $\mathbf{A} \mathbf{d} \mathbf{v}_{\mathcal{H}}^{\text {sec }}$ are of the order $O\left(\frac{q}{2^{n}}+\frac{q^{3}}{2^{l-m}}\right)$. Note that the collision resistance bound is optimal for the $n=256$ variant.

3.9. The Keccak hash function [9] is a chop-MD construction. The compression function $f$ is permutation based. The hash function output is obtained by chopping off $l-n$ bits of the stat€9 Notice that the parameters of Keccak satisfy $l=2 n+m$.

Security of Keccak. The compression function of Keccak is based on one permutation, and collisions and preimages for the compression function can be found in one query to the permutation [15. The Keccak hash function is proven indifferentiable from a random oracle if the underlying permutation is assumed to be ideal [8]. Using Thm. 2, this indifferentiability bound additionally renders an optimal collision resistance bound for Keccak, $\mathbf{A d v}_{H}^{\mathrm{col}}=\Theta\left(q^{2} / 2^{n}\right)$, as well as an optimal preimage second preimage resistance bound $\Theta\left(q / 2^{n}\right)$.

3.10. The Luffa hash function 22 is a chop-MD construction, with a final transformation before chopping. The compression function $f$ is permutation based, and the final transformation $g$ is built on this compression function and a linear function $L^{\prime}$ that chops the state in blocks of length $m$ bits, and XORs these. The compression function employs a linear function $L$ for message injection (MI of [22] ${ }^{10}$. Notice that the state size of Luffa satisfies $l=w \cdot m$.

Security of Luffa. The compression function of Luffa is based on $w$ permutations executed independently. As a consequence, collisions and preimages for the compression function can be found in at most 5 queries to the permutations [15. The Luffa hash function borrows characteristics from the sponge design, if the permutation $P$ consisting of the $w$ permutations $P_{i}$ is considered ideal, and ideas from the indifferentiability proof of [8] may carry over. However, for the case of $w$ different permutations $P_{i}$ this is not immediately clear.

3.11. The Shabal hash function [19] is a chop-MD construction. The message blocks are accompanied with a counter, and the last block is iterated three times. In particular, the function employs a suffix- and prefix-free padding rule. The compression function $f$ is block cipher based 11 . Notice that the parameters of Shabal satisfy $l=384+2 m$.

Security of Shabal. A bound on the collision resistance of the compression function of Shabal is derived in [19]. Concretely, it is proven that the Shabal compression function is collision resistant up to $q=2^{(l-m) / 2}$ queries. Thm. 1 applies to Shabal. Collision and preimage resistance of Shabal are studied in [19], yielding optimal bounds $\mathbf{A} \mathbf{d} \mathbf{v}_{H}^{\text {pre }}=\Theta\left(q / 2^{n}\right)$ and $\mathbf{A d v}_{H}^{\text {col }}=\Theta\left(q^{2} / 2^{n}\right)$. Furthermore, the same authors prove the Shabal hash function to be indifferentiable from a random oracle if the underlying block cipher is assumed to be ideal [19. Using Thm. 2, this indifferentiability bound additionally renders an improved upper bound $O\left(\frac{q}{2^{n}}+\frac{q^{2}}{2^{l-m}}\right)$ on the second preimage resistance. Note that this bound is optimal for the $n=256$ variant.

\footnotetext{
${ }^{9}$ We notice that sponge functions are designed more general [10, but for Keccak this description suffices.

${ }^{10}$ We defined the output transformation in a slightly more complicated but unified way. Essentially, Luffa 256 simply outputs $L^{\prime}(h)$. Observe that we implicitly captured the extra compression function call in the adjusted padding.

${ }^{11}$ Essentially, it is a permutation tweaked by a 1024-bit key, which we view here as a block cipher.
} 
3.12. The SHAvite-3 hash function [14] is a HAIFA construction. The message blocks are accompanied with a HAIFA-counter, and more generally, the function employs a suffix- and prefix-free padding rule. The compression function $f$ is block cipher based.

Security of SHAvite-3. The compression function of SHAvite-3 is the PGV5 compression function, and the security results of [16] carry over. As a consequence, we obtain optimal security bounds on the compression function. The mode of operation of SHAvite-3 is based on the HAIFA structure, and as a consequence all security properties regarding this type hold [13]. In particular, the design preserves collision resistance, and as a consequence we obtain $\mathbf{A d v}_{\mathcal{H}}^{\text {col }}=\Theta\left(q^{2} / 2^{n}\right)$. Also, the design is secure against second preimage attacks. Finally, the SHAvite-3 hash function is indifferentiable from a random oracle if the underlying block cipher is assumed to be ideal, due to the prefix-free padding [20]. This result has been improved under the assumption that the underlying compression function is ideal [11]. However, the compression function of SHAvite-3 is easily differentiable from a random oracle due to the presence of fixed-points.

3.13. The SIMD hash function [38] is a chop-MD construction, with a final transformation before chopping. The hash function employs a suffix-free padding rule. The compression function $f$ is block cipher based, but the last round is executed with a compression function $g$ based on a block cipher $\tilde{E}$ which differs from $E$ in the parametrization. These function employ a quasi-group operation ${ }^{12}$ $L$ [38].

Security of SIMD. The compression function of SIMD is a 'rate-1 Type-I' compression function in the categorization of [49]. Therefore, the results of [49, Thm. 6] carry over, yielding optimal security bounds for the compression function. Observe that these bounds also apply to the function $g$. Observe moreover that these results can easily be adjusted to obtain bound $\mathbf{A} \mathbf{d v}_{\text {chop }}^{\text {col }}=\Theta\left(q^{2} / 2^{n}\right)$. Thm. 1 applies to SIMD, and as a consequence we obtain $\mathbf{A d v}_{\mathcal{H}}^{\text {col }}=\Theta\left(q^{2} / 2^{n}\right)$. Furthermore, the SIMD hash function would be indifferentiable from a random oracle if the underlying compression functions are assumed to be ideal, due to the chopping function at the end [20]. However, the compression functions of SIMD are easily differentiable from a random oracle [17], and we cannot directly apply the results of $[20$.

3.14. The Skein hash function [25] is a chop-MD construction. The message blocks are accompanied with a round-specific tweak 13 and more generally, the function employs a suffix- and prefix-free padding rule. The compression function $f$ is based on a tweakable block cipher.

Security of Skein. The compression function of Skein is the PGV1 compression function, with a difference that a tweak is involved. As claimed in [5], the results of [16] carry over, which in turn results in optimal security bounds on the compression function. Thm. 1 applies to Skein, and as a consequence we obtain $\mathbf{A d v}_{\mathcal{H}}^{\text {col }}=\Theta\left(q^{2} / 2^{n}\right)$. Furthermore, the Skein hash function is proven indifferentiable from a random oracle if the underlying tweakable block cipher is assumed to be ideal [5]. This proof is based on the preimage-awareness approach [24].

\footnotetext{
${ }^{12}$ For any of the variables fixed, the function $L$ is a permutation.

${ }^{13}$ More formally, the design is based on the UBI (unique block identifier) chaining mode which queries its underlying tweakable block cipher on additional tweaks, that differ in each iteration. The general description of Skein involves a specific final transformation. In the primary proposal of the hash function, however, this final transformation consists of another execution of the compression function, with an output-specific tweak and with message $0^{m}$. As we included this final message block in the padding, the given description of Skein suffices.
} 


\section{Summary and Conclusions}

In this survey, we compared the security achieved by the remaining round 2 SHA-3 hash function candidates, when their underlying primitives are assumed to be ideal. The main contribution of this paper is the summary of the security reductions for the hash function candidates in Table 1 . Before giving an interpretation of these results, we first make some remarks on the provided classification.

- Assuming ideality of the underlying primitives (permutations or block ciphers) is not realistic. In particular, none of the candidates' primitives is ideal, and some even have identified weaknesses. However, assuming ideality of these primitives gives significantly more confidence in the security of the higher level structure and is the only way to get useful (and comparable) security bounds on the candidate hash functions;

- The fact that different hash functions have different bounds, does not directly imply that one of the functions offers a higher level of security: albeit the underlying structure of the basic primitives is abstracted away (see the previous item), still many differences among the schemes remain (chaining size, message input size, etc.). Moreover, not all bounds are tight.

Security of the compression function. For the sponge(-like) hash functions, CubeHash, Fugue, $\mathrm{JH}$, Keccak and Luffa, collisions and preimages for the compression function can be found in a constant number of queries. This fact does not have direct implications for the security of the hash function. In fact, the only consequence is that it becomes unreasonable to assume ideality of the compression function in order to prove security at a higher level. Most of the remaining nine candidates are provided with a tight bound for collision and/or preimage resistance of the compression function, merely due to the results of [1649. Single exceptions are BLAKE and BMW, for which the results of [49] are not directly applicable. No security results are known for the second preimage resistance of the nine remaining candidates: albeit collision resistance implies second preimage resistance [46], the obtained security bounds would be below the requirements of NIST [43;

Indifferentiability of the hash function. Eight of the candidates are proven indifferentiable from a random oracle, and six of the candidates have a similar constructions to ones proven indifferentiable. We note that there exist some differences among the bounds. For instance, for the hash function variant outputting $n=512$ bits, the indifferentiability bounds are varying between $O\left(K q^{3} / 2^{512}\right)$ and $O\left((K q)^{2} / 2^{1024}\right)$. These differences are mainly caused by the fact that the bounds are parameterized by the internal chaining value size $l$, rather than the output size $n$ (as is the case for bounds on the collision resistance). As a consequence, a higher state size often results in a better indifferentiability bound. The indifferentiability results are powerful, as they render bounds on the (second) preimage and collision resistance of the design (Thm. 2);

(Second) preimage resistance of the hash function. Most of the hash functions are not provided with an optimal security bound on the preimage and second preimage resistance. Main cause for this is that the MD design does not preserve (second) preimage resistance [2]. Additionally, the (second) preimage bound that can be derived via the indifferentiability (Thm. 2 ) is not always sufficiently tight. Proving security against these attacks could be attempted either by making a different (possibly weaker) assumption on the compression function or by basing it directly on the ideality of the underlying block cipher or permutation(s). We notice that a fruitful direction might be the graph based approach followed by the designers of Shabal [19];

Collision resistance of the hash function. Except for the sponge(-like) functions, the collision resistance preservation result of Thm. 1 (App. A) applies to all candidates. This theorem results in a bound on the generic collision resistance of the hash function, which, intuitively, means that 'finding collisions for the hash function is at least as hard as finding collisions for (one of) 
the underlying function(s)'. Together with the collision resistance bounds on the compression functions in the ideal model, the preservation result allows for obtaining a collision resistance bound on the entire hash function. This leads to optimal bounds on the collision resistance for ECHO, Grøstl, Hamsi, SHAvite-3, SIMD and Skein. For BLAKE, CubeHash, JH (for $n=$ 256), Keccak and Shabal, the same optimal bound is obtained differently (e.g. based on the indifferentiability of the hash function). Again, the graph based approach may be suitable to prove collision resistance of the candidates for which no collision resistance bound is yet obtained.

A hash function that is provided with a sound security analysis, is not necessarily a 'good' function, nor is it a 'bad' function if only little security results are known. The quality of the hash function depends further on other criteria not covered in this classification, such as the strength of the basic underlying primitives and software/hardware performance. Yet, security reductions guarantee that the hash function has no severe structural weaknesses, and in particular that the design does not suffer weaknesses that can be trivially exploited by cryptanalysts. Therefore, we see the provided security analysis as a fair comparison of the SHA-3 candidates and an important contribution to the selection of the finalists. To the best of our knowledge, we included all security results to date. However, we welcome suggestions, remarks or information about provable security results that could improve the quality of this work.

ACKnowledgments. This work has been funded in part by the IAP Program P6/26 BCRYPT of the Belgian State (Belgian Science Policy), and in part by the European Commission through the ICT program under contract ICT-2007-216676 ECRYPT II. The first author is supported by a Ph.D. Fellowship from the Flemish Research Foundation (FWO-Vlaanderen). The second author is supported by a Ph.D. Fellowship from the Institute for the Promotion of Innovation through Science and Technology in Flanders (IWT-Vlaanderen).

We would like to thank Joan Daemen, Praveen Gauravaram, Charanjit Jutla and Christian Rechberger for the helpful comments.

\section{References}

1. Andreeva, E., Mennink, B., Preneel, B.: On the indifferentiability of the Grøstl hash function. In: SCN '10. LNCS, vol. 6280, pp. 88-105. Springer-Verlag, Berlin (2010)

2. Andreeva, E., Neven, G., Preneel, B., Shrimpton, T.: Seven-property-preserving iterated hashing: ROX. In: ASIACRYPT '07. LNCS, vol. 4833, pp. 130-146. Springer-Verlag, Berlin (2007)

3. Aumasson, J.P., Henzen, L., Meier, W., Phan, R.: SHA-3 proposal BLAKE (2009)

4. Bellare, M., Canetti, R., Krawczyk, H.: Keying hash functions for message authentication. In: CRYPTO '96. LNCS, vol. 1109, pp. 1-15. Springer-Verlag, Berlin (1996)

5. Bellare, M., Kohno, T., Lucks, S., Ferguson, N., Schneier, B., Whiting, D., Callas, J., Walker, J.: Provable security support for the skein hash family (2009)

6. Benadjila, R., Billet, O., Gilbert, H., Macario-Rat, G., Peyrin, T., Robshaw, M., Seurin, Y.: SHA-3 Proposal: ECHO (2009)

7. Bernstein, D.: CubeHash specification (2009)

8. Bertoni, G., Daemen, J., Peeters, M., Van Assche, G.: On the indifferentiability of the sponge construction. In: EUROCRYPT '08. LNCS, vol. 4965, pp. 181-197. Springer-Verlag, Berlin (2008)

9. Bertoni, G., Daemen, J., Peeters, M., Van Assche, G.: The KECCAK sponge function family (2009)

10. Bertoni, G., Daemen, J., Peeters, M., Van Assche, G.: Sponge functions (ECRYPT Hash Workshop 2007)

11. Bhattacharyya, R., Mandal, A., Nandi, M.: Indifferentiability characterization of hash functions and optimal bounds of popular domain extensions. In: INDOCRYPT '09. LNCS, vol. 5922, pp. 199-218. Springer-Verlag, Berlin (2009)

12. Bhattacharyya, R., Mandal, A., Nandi, M.: Security analysis of the mode of JH hash function. In: FSE '10. LNCS, vol. 6147, pp. 168-191. Springer-Verlag, Berlin (2010) 


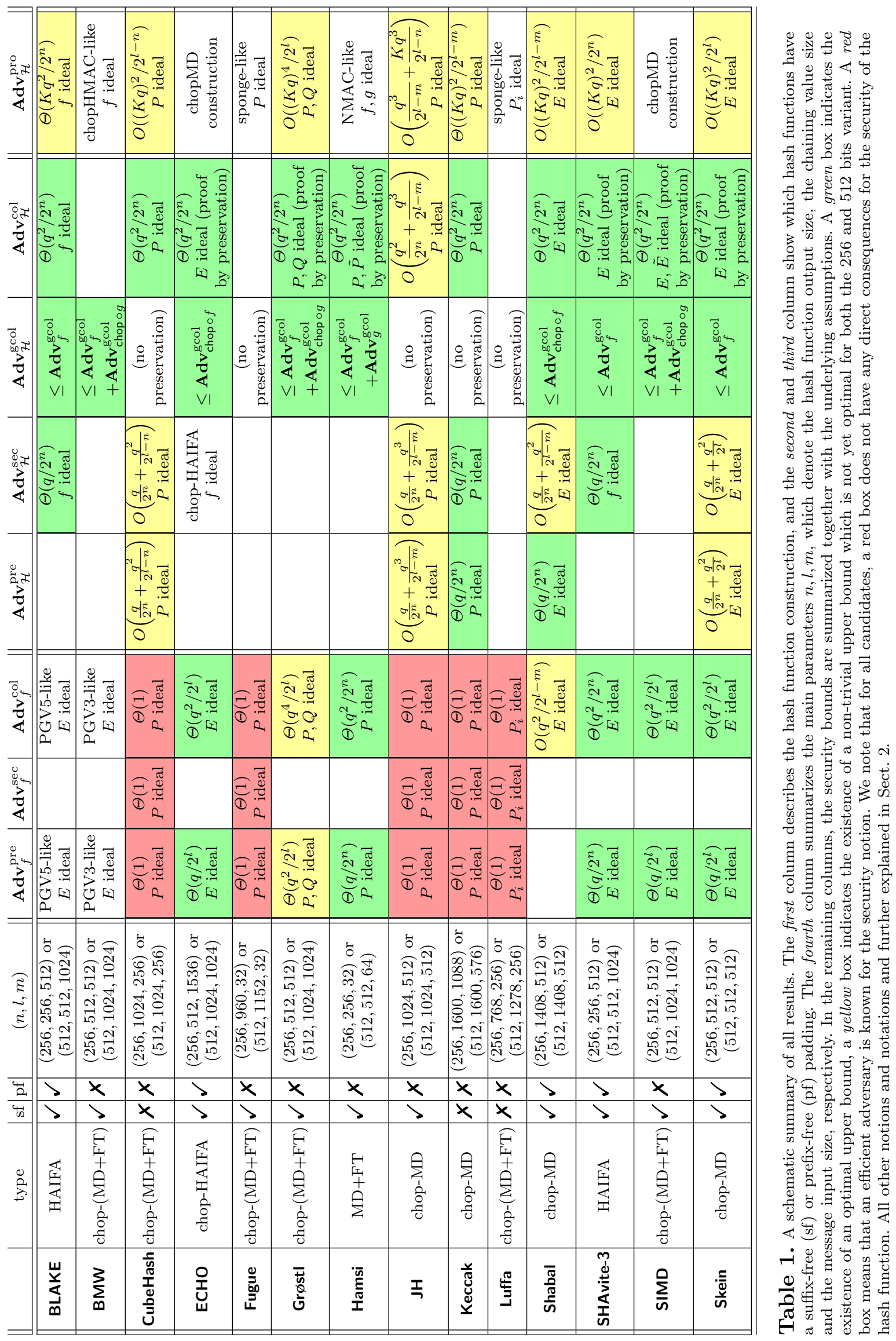


13. Biham, E., Dunkelman, O.: A framework for iterative hash functions - HAIFA. Cryptology ePrint Archive, Report $2007 / 278$ (2007)

14. Biham, E., Dunkelman, O.: The SHAvite-3 Hash Function (2009)

15. Black, J., Cochran, M., Shrimpton, T.: On the impossibility of highly-efficient blockcipher-based hash functions. In: EUROCRYPT '05. LNCS, vol. 3494, pp. 526-541. Springer-Verlag, Berlin (2005)

16. Black, J., Rogaway, P., Shrimpton, T.: Black-box analysis of the block-cipher-based hash-function constructions from PGV. In: CRYPTO '02. LNCS, vol. 2442, pp. 320-335. Springer-Verlag, Berlin (2002)

17. Bouillaguet, C., Fouque, P.A., Leurent, G.: Security analysis of SIMD (2010)

18. Bouillaguet, C., Fouque, P.A., Shamir, A., Zimmer, S.: Second preimage attacks on dithered hash functions. Cryptology ePrint Archive, Report 2007/395 (2007)

19. Bresson, E., Canteaut, A., Chevallier-Mames, B., Clavier, C., Fuhr, T., Gouget, A., Icart, T., Misarsky, J.F., Naya-Plasencia, M., Paillier, P., Pornin, T., Reinhard, J.R., Thuillet, C., Videau, M.: Shabal, a Submission to NIST's Cryptographic Hash Algorithm Competition (2009)

20. Coron, J.S., Dodis, Y., Malinaud, C., Puniya, P.: Merkle-Damgård revisited: How to construct a hash function. In: CRYPTO '05. LNCS, vol. 3621, pp. 430-448. Springer-Verlag, Berlin (2005)

21. Damgård, I.: A design principle for hash functions. In: CRYPTO '89. LNCS, vol. 435, pp. 416-427. SpringerVerlag, Berlin (1990)

22. De Cannière, C., Sato, H., Watanabe, D.: Hash Function Luffa (2009)

23. Dodis, Y., Puniya, P.: Getting the best out of existing hash functions; or what if we are stuck with SHA? In: ACNS '08. LNCS, vol. 5037, pp. 156-173. Springer-Verlag, Berlin (2008)

24. Dodis, Y., Ristenpart, T., Shrimpton, T.: Salvaging merkle-damgård for practical applications. In: EUROCRYPT '09. LNCS, vol. 5479, pp. 371-388. Springer-Verlag, Berlin (2009)

25. Ferguson, N., Lucks, S., Schneier, B., Whiting, D., Bellare, M., Kohno, T., Callas, J., Walker, J.: The Skein Hash Function Family (2009)

26. Ferguson, N., Lucks, S., Schneier, B., Whiting, D., Bellare, M., Kohno, T., Callas, J., Walker, J.: Engineering comparison of SHA-3 candidates (2010)

27. Fleischmann, E., Forler, C., Gorski, M.: Classification of the SHA-3 candidates. Cryptology ePrint Archive, Report 2008/511 (2008)

28. Fouque, P.A., Stern, J., Zimmer, S.: Cryptanalysis of tweaked versions of SMASH and reparation. In: SAC '08. LNCS, vol. 5381, pp. 136-150. Springer-Verlag, Berlin (2009)

29. Gauravaram, P., Bagheri, N.: ECHO compression function is not indifferentiable from a FIL-RO (2010)

30. Gauravaram, P., Knudsen, L., Matusiewicz, K., Mendel, F., Rechberger, C., Schläffer, M., Thomsen, S.: Grøstl a SHA-3 candidate (2009)

31. Gligoroski, D., Klima, V., Knapskog, S.J., El-Hadedy, M., Amundsen, J., Mjølsnes, S.F.: Cryptographic Hash Function BLUE MIDNIGHT WISH (2009)

32. Halevi, S., Hall, W., Jutla, C.: The Hash Function "Fugue" (2009)

33. Halevi, S., Krawczyk, H.: Strengthening digital signatures via randomized hashing. In: CRYPTO '06. LNCS, vol. 4117, pp. 41-59. Springer-Verlag, Berlin (2006)

34. Kelsey, J., Schneier, B.: Second preimages on n-bit hash functions for much less than $2^{\mathrm{n}}$ work. In: EUROCRYPT '05. LNCS, vol. 3494, pp. 474-490. Springer-Verlag, Berlin (2005)

35. Kobayashi, K., Ikegami, J., Matsuo, S., Sakiyama, K., Ohta, K.: Evaluation of hardware performance for the SHA-3 candidates using SASEBO-GII. Cryptology ePrint Archive, Report 2010/010 (2010)

36. Küçük, Ö.: The Hash Function Hamsi (2009)

37. Lai, X., Massey, J.: Hash function based on block ciphers. In: EUROCRYPT '92. LNCS, vol. 658, pp. 55-70. Springer-Verlag, Berlin (1992)

38. Leurent, G., Bouillaguet, C., Fouque, P.A.: SIMD is a Message Digest (2009)

39. Lucks, S.: A failure-friendly design principle for hash functions. In: ASIACRYPT '05. LNCS, vol. 3788, pp. 474-494. Springer-Verlag, Berlin (2005)

40. Maurer, U., Renner, R., Holenstein, C.: Indifferentiability, impossibility results on reductions, and applications to the random oracle methodology. In: TCC '04. LNCS, vol. 2951, pp. 21-39. Springer-Verlag, Berlin (2004)

41. Merkle, R.: One way hash functions and DES. In: CRYPTO '89. LNCS, vol. 435, pp. 428-446. Springer-Verlag, Berlin (1990)

42. Nandi, M.: Characterizing padding rules of MD hash functions preserving collision security. In: ACISP '09. LNCS, vol. 5594, pp. 171-184. Springer-Verlag, Berlin (2009)

43. National Institute for Standards and Technology. Announcing Request for Candidate Algorithm Nominations for a New Cryptographic Hash Algorithm (SHA3) Family (November 2007)

44. Preneel, B., Govaerts, R., Vandewalle, J.: Hash functions based on block ciphers: A synthetic approach. In: CRYPTO '93. LNCS, vol. 773, pp. 368-378. Springer-Verlag, Berlin (1993)

45. Rogaway, P.: Formalizing human ignorance. In: VIETCRYPT '06. LNCS, vol. 4341, pp. 211-228. Springer-Verlag, Berlin (2006) 
46. Rogaway, P., Shrimpton, T.: Cryptographic hash-function basics: Definitions, implications, and separations for preimage resistance, second-preimage resistance, and collision resistance. In: FSE '04. LNCS, vol. 3017, pp. 371388. Springer-Verlag, Berlin (2004)

47. Rogaway, P., Steinberger, J.: Security/efficiency tradeoffs for permutation-based hashing. In: EUROCRYPT '08. LNCS, vol. 4965, pp. 220-236. Springer-Verlag, Berlin (2008)

48. Stam, M.: Beyond uniformity: Better security/efficiency tradeoffs for compression functions. In: CRYPTO '08. LNCS, vol. 5157, pp. 397-412. Springer-Verlag, Berlin (2008)

49. Stam, M.: Blockcipher-based hashing revisited. In: FSE '09. LNCS, vol. 5665, pp. 67-83. Springer-Verlag, Berlin (2009)

50. Tillich, S., Feldhofer, M., Kirschbaum, M., Plos, T., Schmidt, J.M., Szekely, A.: High-speed hardware implementations of BLAKE, Blue Midnight Wish, CubeHash, ECHO, Fugue, Grøstl, Hamsi, JH, Keccak, Luffa, Shabal, SHAvite-3, SIMD, and Skein. Cryptology ePrint Archive, Report 2009/510 (2009)

51. Wang, X., Yin, Y.L., Yu, H.: Finding collisions in the full SHA-1. In: CRYPTO '05. LNCS, vol. 3621, pp. 17-36. Springer-Verlag, Berlin (2005)

52. Wang, X., Yu, H.: How to break MD5 and other hash functions. In: EUROCRYPT '05. LNCS, vol. 3494, pp. 19-35. Springer-Verlag, Berlin (2005)

53. Wu, H.: The Hash Function JH (2009)

\section{A Preservation of Collision Resistance}

For the purpose of the analysis of the SHA-3 candidates, we generalize the well-known result by Merkle and Damgård. The result of Thm. 1 differs in three cases: we consider any suffix-free padding, the proof allows for different compression functions in one hash function evaluation, and it includes an optional chopping at the end. Related work can, a.o., be found in [4121|23|42].

Theorem 1. Let $l, m, n \in \mathbb{N}$ such that $l \geq n$. Let pad $: \mathbb{Z}_{2}^{*} \rightarrow\left(\mathbb{Z}_{2}^{m}\right)^{*}$ be a suffix-free padding and let $f, g: \mathbb{Z}_{2}^{l} \times \mathbb{Z}_{2}^{m} \rightarrow \mathbb{Z}_{2}^{l}$ be two compression functions. Consider the hash function $\mathcal{H}: \mathbb{Z}_{2}^{*} \rightarrow \mathbb{Z}_{2}^{n}$ defined as follows (cf. Fig. 2), where $h_{0}=$ IV is the initialization vector:

$$
\begin{aligned}
\mathcal{H}(M)=h, \text { where: } & \left(M_{1}, \ldots, M_{k}\right)=\operatorname{pad}(M), \\
& h_{i}=f\left(h_{i-1}, M_{i}\right) \text { for } i=1, \ldots, k-1, \\
& h_{k}=g\left(h_{k-1}, M_{k}\right), \\
& h=\operatorname{chop}_{l-n}\left(h_{k}\right) .
\end{aligned}
$$

Define the function $g^{\prime}: \mathbb{Z}_{2}^{l} \times \mathbb{Z}_{2}^{m} \rightarrow \mathbb{Z}_{2}^{n}$ by $g^{\prime}=$ chop $_{l-n} \circ g$. Then, the advantage of finding collisions for $\mathcal{H}$ is upper bounded by the advantage of finding collisions for $f$ or $g^{\prime}$. Formally, if $f$ is $\left(t_{1}, \varepsilon_{1}\right)$ collision secure, and $g^{\prime}$ is $\left(t_{2}, \varepsilon_{2}\right)$ collision secure, then $\mathcal{H}$ is $(t, \varepsilon)$ collision secure for $\varepsilon=\varepsilon_{1}+\varepsilon_{2}$, and $t=\min \left\{t_{1}, t_{2}\right\}-2(K-1) \tau_{f}$, where $\tau_{f}$ is the time to evaluate $f$ and $K$ is the maximum length of the messages, in blocks.

Proof. Suppose $\mathcal{A}$ is a $(t, \varepsilon)$ collision finding attacker for $\mathcal{H}$. We construct collision finding adversaries $\mathcal{B}_{1}$ and $\mathcal{B}_{2}$ for $f$ and $g$, respectively, using the following observation.

Let $M, M^{\prime}$ be two distinct messages such that $\mathcal{H}(M)=\mathcal{H}\left(M^{\prime}\right)$. Let $\left(M_{1}, \ldots, M_{k}\right)$ be the padded message of $M$, and $\left(M_{1}^{\prime}, \ldots, M_{k^{\prime}}^{\prime}\right)$ be the padded message of $M^{\prime}$. Define the intermediate state values $h_{i}, h_{i}^{\prime}$ similarly. A collision on $M, M^{\prime}$ means that $\operatorname{chop}_{l-n}\left(g\left(h_{k-1}, M_{k}\right)\right)=\operatorname{chop}_{l-n}\left(g\left(h_{k^{\prime}-1}^{\prime}, M_{k^{\prime}}^{\prime}\right)\right)$. Now, if $\left(h_{k-1}, M_{k}\right) \neq\left(h_{k^{\prime}-1}^{\prime}, M_{k^{\prime}}^{\prime}\right)$ this results in a collision for $g^{\prime}$. Assume the contrary, and let $j \in\left\{1, \ldots, \min \left\{k, k^{\prime}\right\}-1\right\}$ be the minimal index such that $\left(h_{k-j-1}, M_{k-j}\right) \neq\left(h_{k^{\prime}-j-1}^{\prime}, M_{k^{\prime}-j}^{\prime}\right)$. We notice that such index $j$ exists: in case $k=k^{\prime}$ it exists as $M \neq M^{\prime}$, and in case $k \neq k^{\prime}$ it exists as the padding rule is suffix-free. By definition of the index $j$, we have $h_{k-j}=h_{k^{\prime}-j}^{\prime}$, and in particular we obtain a collision for $f$ :

$$
f\left(h_{k-j-1}, M_{k-j}\right)=h_{k-j}=h_{k^{\prime}-j}^{\prime}=f\left(h_{k^{\prime}-j-1}^{\prime}, M_{k^{\prime}-j}^{\prime}\right) .
$$


Both $\mathcal{B}_{1}, \mathcal{B}_{2}$ follow this procedure. If $M, M^{\prime}$ define a collision for $f, \mathcal{B}_{1}$ outputs this collision. Similarly for $\mathcal{B}_{2}$ and $g^{\prime}$. Both adversaries work in time at most $t+2(K-1) \tau_{f}$, from which we deduce $t \geq \min \left\{t_{1}, t_{2}\right\}-2(K-1) \tau_{f}$. The messages $M, M^{\prime}$ define a collision for $f$ or $g^{\prime}$. Thus, we obtain $\varepsilon \leq \varepsilon_{1}+\varepsilon_{2}$.

In case the design is based on the compression function $f$ only (but it may still include the chopping), the above result can easily be simplified to $\operatorname{Adv}_{\mathcal{H}}^{\text {gcol }}(\mathcal{A}) \leq \mathbf{A d v}_{f^{\prime}}^{\text {gcol }}\left(\mathcal{B}_{1}\right)$, where $f^{\prime}$ is defined by $f^{\prime}=\operatorname{chop}_{l-n} \circ f$. Observe that this result also holds if $l=n$, and in particular, the basic theorems of Merkle and Damgård are covered as well. We note that Thm. 1 can be generalized arbitrarily, e.g. to more different compression functions, but for the purpose of this paper, the mentioned generalization of the Merkle-Damgård structure suffices.

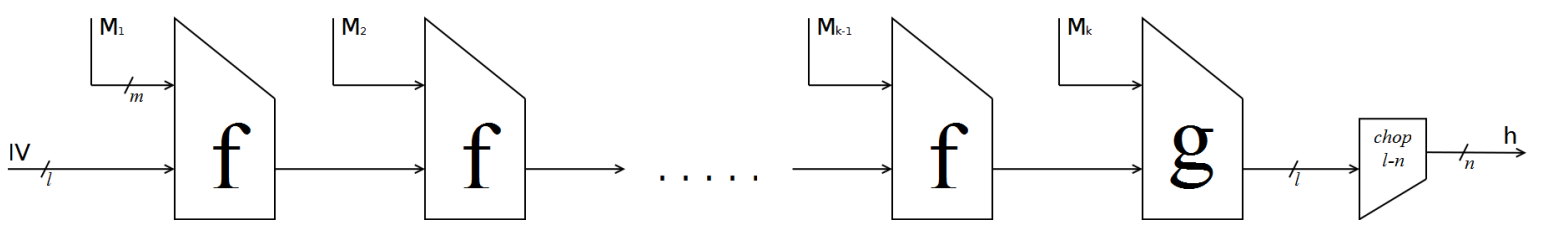

Fig. 2. A generalized Merkle-Damgård structure. $f, g$ are two compression functions, and chop $_{l-n}$ chops off $l-n$ bits of the state.

\section{B Security Implications of Indifferentiability}

Indifferentiability assures that a design is structurally correct, and that it can replace a random oracle without security loss. In particular, it guarantees that, up to a certain degree, the design is secured against any generic attack, like finding preimages, collisions, multicollisions, etc. In Thm. 2 , we formally prove a security reduction to derive security against specific attacks from the indifferentiability of a hash function. This proof is based on a personal communication with Joan Daemen.

Theorem 2. Let $\mathcal{H}$ be a hash function, built on underlying primitive $\pi$, and $R O$ be a random oracle, where $\mathcal{H}$ and $R O$ have the same domain and range space. Denote by $\boldsymbol{A d v}_{\mathcal{H}}^{\text {pro }}(q)$ the advantage of distinguishing $(\mathcal{H}, \pi)$ from $(R O, S)$, for some simulator $S$, maximized over all distinguishers $\mathcal{D}$ making at most $q$ queries. Let atk be a security property of $\mathcal{H}$. Denote by $\boldsymbol{A d v}_{\mathcal{H}}^{\text {atk }}(q)$ the advantage of breaking $\mathcal{H}$ under atk, maximized over all adversaries $\mathcal{A}$ making at most q queries. Then:

$$
\boldsymbol{A} \boldsymbol{d} \boldsymbol{v}_{\mathcal{H}}^{\mathrm{atk}}(q) \leq \boldsymbol{P} \boldsymbol{r}_{R O}^{\mathrm{atk}}(q)+\boldsymbol{A} \boldsymbol{d} \boldsymbol{v}_{\mathcal{H}}^{\mathrm{pro}}(q)
$$

where $\boldsymbol{P r}_{R O}^{\mathrm{atk}}(q)$ denotes the success probability of a generic attack against $\mathcal{H}$ under atk, after at most q queries.

Proof. Let $\mathcal{A}$ be any $(q, \varepsilon)$ attacker for $\mathcal{H}$ under security notion atk, and assume $\varepsilon>\mathbf{P r}_{R O}^{\text {atk }}(q)$. We define a distinguisher $\mathcal{D}$ for the indifferentiability of $\mathcal{H}$ as follows: $\mathcal{D}$ makes the same queries as $\mathcal{A}$, and obtains a query history $Q$ (with query answers coming from either $\mathcal{H}$ or $R O$ ). Next, $\mathcal{D}$ outputs 1 if $Q$ violates security notion atk, and 0 otherwise. Denote by $\operatorname{Adv}_{\mathcal{H}}^{\text {pro }}(\mathcal{D})$ the success probability of $\mathcal{D}$. By definition, we have $\left|\operatorname{Pr}\left(\mathcal{D}^{\mathcal{H}, \pi}=1\right)-\operatorname{Pr}\left(\mathcal{D}^{R O, S}=1\right)\right|=\mathbf{A d v}_{\mathcal{H}}^{\text {pro }}(\mathcal{D}) \leq \mathbf{A d v}_{\mathcal{H}}^{\text {pro }}(q)$. By $E_{\mathcal{H}}\left(\right.$ resp. $\left.E_{R O}\right)$, we denote the event that $Q$ defines a set of query pairs that break $\mathcal{H}$ (resp. $R O$ ) 
under security notion atk. The distinguisher outputs 1 if and only if $Q$ violates security notion atk, and hence we obtain:

$$
\begin{aligned}
\operatorname{Pr}\left(\mathcal{D}^{\mathcal{H}, \pi}=1\right) & =\operatorname{Pr}\left(\mathcal{D}^{\mathcal{H}, \pi}=1 \mid E_{\mathcal{H}}\right) \operatorname{Pr}\left(E_{\mathcal{H}}\right)+\operatorname{Pr}\left(\mathcal{D}^{\mathcal{H}, \pi}=1 \mid \neg E_{\mathcal{H}}\right) \operatorname{Pr}\left(\neg E_{\mathcal{H}}\right) \\
& =1 \cdot \operatorname{Pr}\left(E_{\mathcal{H}}\right)+0=\varepsilon .
\end{aligned}
$$

Similarly, we get $\operatorname{Pr}\left(\mathcal{D}^{R O, S}=1\right)=\operatorname{Pr}\left(E_{R O}\right)=\mathbf{P r}_{R O}^{\text {atk }}(q)$. As $\varepsilon>\operatorname{Pr}_{R O}^{\text {atk }}(q)$, we consequently derive $\varepsilon \leq \mathbf{P r}_{R O}^{\text {atk }}(q)+\mathbf{A d v}_{\mathcal{H}}^{\text {pro }}(q)$. This holds for any $(q, \varepsilon)$ adversary for $\mathcal{H}$ under security notion atk, which completes the proof.

\section{Padding Rules}

The padding rules of all SHA-3 hash function candidates are summarized. All padding functions output bit strings parsed as sequences of $m$-bit blocks, where $m$ is the message block length of the corresponding function. Formally, for each candidate, for $n \in\{256,512\}$ the padding function pad : $\mathbb{Z}_{2}^{*} \rightarrow\left(\mathbb{Z}_{2}^{m}\right)^{*}$ is defined as follows. For the hash functions BLAKE, ECHO, Shabal, SHAvite-3 and Skein, the complete padding rule of the corresponding hash function is additionally defined by a counter or tweak (as explained in Sect. 3). Particularly, all hash functions employ an injective padding rule.

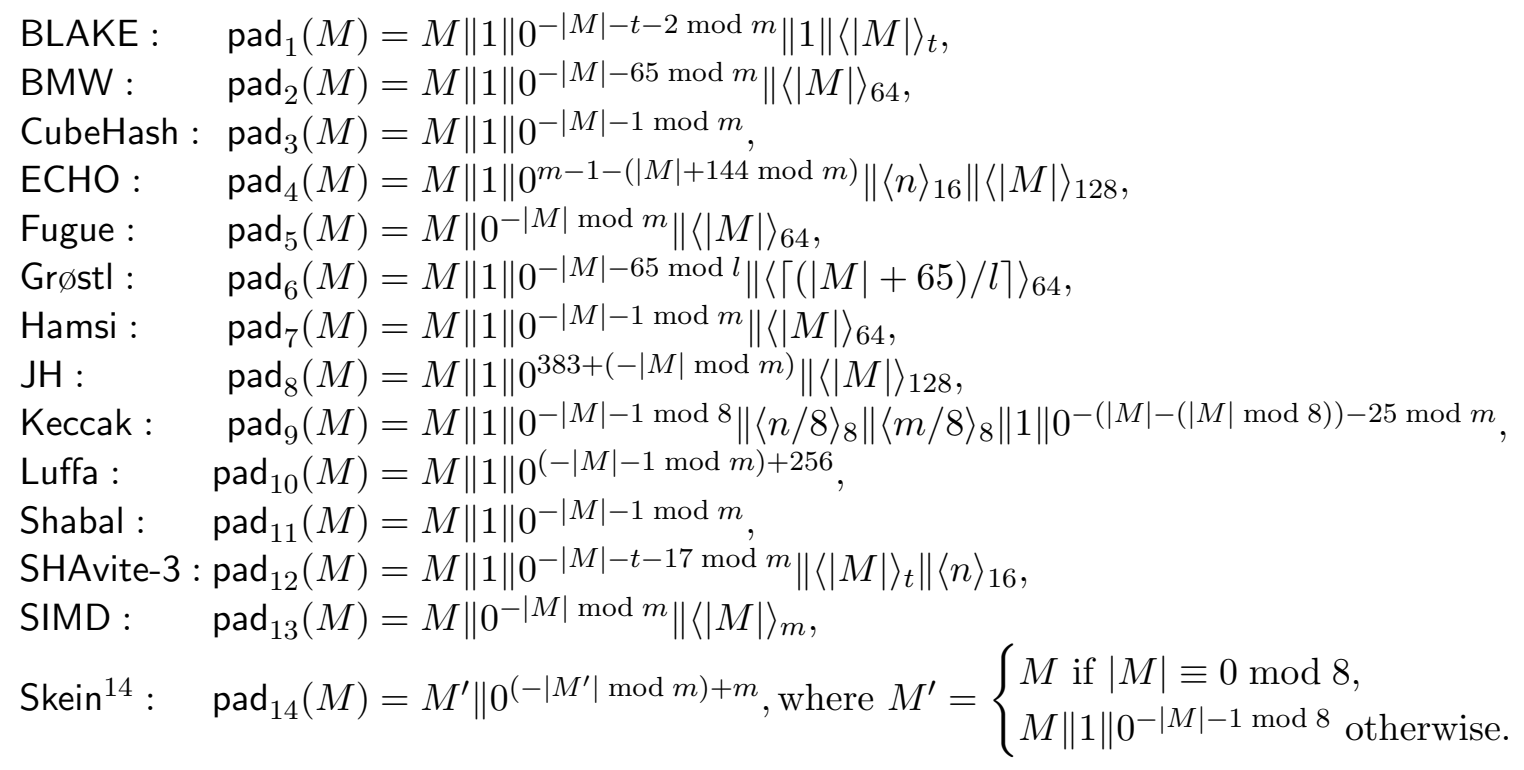

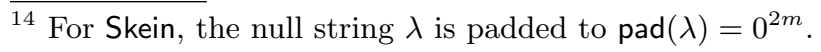

